\title{
Meningioma microcístico com metástase pulmonar em canino: relato de caso
}

[Microcystic meningioma with pulmonary metastasis in canine: case report]

R. Zamboni ${ }^{1}$, T.S. Alberti ${ }^{1}$, H.V. Schied ${ }^{1}$, C.S. Bermann ${ }^{2}$, C.B. Brunner ${ }^{3}$, F.R. Venancio ${ }^{3}$, E.M.J. Arantes ${ }^{4}$, E.S.V. Sallis ${ }^{5}$, M.B. Raffi ${ }^{5}$

${ }^{1}$ Aluna de pós-graduação - Universidade Federal de Pelotas - Capão do Leão, RS

${ }^{2}$ Aluna de pós-graduação - Instituto de Biologia - Universidade Federal de Pelotas - Capão do Leão, RS

${ }^{3}$ Aluno do Programa de Residência - Universidade Federal de Pelotas - Capão do Leão, RS

${ }^{4}$ Aluna de graduação - Faculdade de Veterinária - Universidade Federal de Pelotas - Capão do Leão, RS

${ }^{5}$ Faculdade de Veterinária - Universidade Federal de Pelotas - Capão do Leão, RS

\section{RESUMO}

Meningiomas são os principais tumores primários do sistema nervoso central (SNC) que afetam cães e gatos. $\mathrm{Na}$ maioria dos casos, são neoplasias benignas, geralmente expansivas, causando compressão do SNC, e raramente fazem metástase para outros órgãos. O presente trabalho tem como objetivo relatar a ocorrência de um meningioma microcístico com metástase pulmonar em um canino de 11 anos de idade, com sinais clínicos de andar cambaleante, compressão da cabeça contra objetos, agitação, salivação e agressividade. Na necropsia, foram observadas, no encéfalo, massas bem delimitadas pardo-avermelhadas, firmes, de aspecto granular, localizadas no córtex parietal e nos núcleos da base. Inúmeras micronodulações de aspecto semelhante foram observadas no pulmão. Histologicamente observaram-se nódulos formados por células neoplásicas fusiformes, com núcleos grandes e alongados e nucléolos evidentes, dispostas de forma frouxa, formando vacúolos e microcistos. À imuno-histoquímica, o meningioma apresentou marcação fortemente positiva para citoqueratina e negativa para vimentina. Por meio da histopatologia e da imuno-histoquímica, foi possível estabelecer a classificação histológica de meningioma microcístico, bem como diferenciá-lo de outras doenças que cursam com sinais nervosos.

Palavras-chave: meningioma microcístico, metástase, pulmão, imuno-histoquímica, sistema nervoso central

\begin{abstract}
Meningiomas are the main tumors of the central nervous system (CNS) affecting dogs and cats. In most of the cases they are benign neoplasms, usually expansive, causing compression of the CNS and rarely metastasize to other organs. We describe the occurrence of a microcystic meningioma with pulmonary metastasis in an 11 year - old canine with clinical signs of staggering gait, head compression against objects, agitation, salivation and aggressiveness. At necropsy, well-defined, firm, granular-looking masses located in the parietal cortex and nuclei of the base were observed in the encephalon. Numerous micronodulations of similar appearance were observed in the lung. Histologically, nodules formed by spindle neoplastic cells with large, elongated nuclei and evident nuclei were loosely arranged, forming vacuoles and microcysts. Immunohistochemistry were strongly positive for cytokeratin and negative for vimentin. Through the histopathology and immunohistochemistry, it was possible to establish the histological classification of microcystic meningioma, as well as to differentiate from other diseases that present with nervous signals.
\end{abstract}

Keywords: microcystic meningioma, metastasis, lung, immunohistochemistry, central nervous system

\section{INTRODUÇÃO}

Meningiomas são tumores mesodérmicos, comumente benignos (Montoliu et al., 2006), sendo as variantes malignas observadas em apenas 0.17/100.000/ano dos casos (Marosi et al., 2008). Em caninos, é a neoplasia

Recebido em 5 de abril de 2019

Aceito em 15 de agosto de 2019

E-mail: rosi_zamboni@yahoo.com.br intracraniana mais frequente. Nessa espécie, na maioria dos casos, apresentam-se como massas solitárias e bem delimitadas, que crescem por expansão compressiva ou infiltração na neurópila subjacente (Higgins et al., 2017). Metástases extracraniais são incomuns, mas podem ser observadas principalmente nos pulmões (Montoliu et al., 2006). Aproximadamente 3,0\% 
das neoplasias que acometem caninos são intracraniais (Montoliu et al., 2006; Snyder et al., 2006; Song et al., 2013). Estima-se que os meningiomas representam de $13 \%$ a $26 \%$ de todos os tumores intracranianos em cães, casuística similar à observada nos humanos (Hicks et al., 2015; Higgins et al., 2017).

Os meningiomas são classificados de acordo com o tipo celular, o comportamento biológico e o padrão topográfico apresentado, podendo ser meningotelial, fibroblástico, transicional, psamomatoso, papilar, microcístico, mixoide, angiomatoso e anaplásico (Higgins et al., 2017). Acometem comumente cães adultos senis com mais de 10 anos de idade (Higgins et al., 2017). Nessa espécie, não há predisposição por sexo, entretanto, em humanos, são mais comuns em mulheres (Montoliu et al., 2006; Marcasso et al., 2015). Entre as raças mais predispostas estão o Boxer e o Golden Retriever (Higgins et al., 2017).

Essa neoplasia é imunorreativa a diversos anticorpos na técnica de imuno-histoquímica, podendo apresentar marcação variada conforme o subtipo tumoral. Entre os anticorpos utilizados estão vimetina, S100, enolase neuronal específica, E-caderina, proteína glial fibrilar ácida e citoqueratina (Montoliu et al., 2006; Higgins et al., 2017). Este trabalho tem como objetivo descrever um caso de meningioma microscístico, com metástase pulmonar, em um canino diagnosticado no Laboratório Regional de Diagnóstico (LRD) da Faculdade de Veterinária da Universidade Federal de Pelotas-UFPel.

\section{RELATO DE CASO}

Foi encaminhado ao Hospital de Clínicas Veterinárias (HCV) da Faculdade de Veterinária (FV) da Universidade Federal de Pelotas (UFPel) um canino, macho, sem raça definida, com 11 anos de idade, apresentando sintomatologia nervosa. Os sinais clínicos caracterizaram-se por andar cambaleante, compressão da cabeça contra objetos, agitação, salivação e agressividade. Devido ao prognóstico desfavorável, os tutores optaram pela eutanásia, sendo o cadáver encaminhado ao Laboratório Regional de Diagnóstico (LRD) da FV/UFPel. Na necropsia, fragmentos dos órgãos das cavidades abdominal e torácica e encéfalo foram coletados e fixados em formalina tamponada a $10 \%$. Após $48 \mathrm{~h}$, os órgãos foram clivados, incluídos em parafina, cortados em secções de $3 \mu \mathrm{m}$ de espessura e corados pela técnica de rotina, hematoxilina e eosina (HE).

Cortes do encéfalo e do pulmão, coletados em lâminas positivadas, foram processados no laboratório de imuno-histoquímica do LRD/FV/UFPel, incubados em overnight durante $16 \mathrm{~h}$ a $4^{\circ} \mathrm{C}$, com anticorpo monoclonal primário anticitoqueratina (clone AE1/AE3, BioCare Medical), na diluição de 1:100, e vimentina (clone V9, BioCare Medical), na diluição 1:100. As imunomarcações foram visualizadas por meio da utilização do 3-3' diaminabenzidina (DAB). As secções foram contracoradas com hematoxilina de Harris. Como controle negativo de cada caso, utilizou-se uma secção do tecido analisado incubado apenas com o diluente do anticorpo (PBST). Tecido cutâneo normal canino foi utilizado nos controles positivos, sendo a epiderme adjacente considerada como controle positivo para a citoqueratina [AE1/AE3], e a derme (fibras de colágeno e endotélio de vasos sanguíneos) para a vimentina.

Macroscopicamente foram observadas, no encéfalo, massas bem delimitadas pardoavermelhadas, firmes, de aspecto granular, localizadas no córtex parietal, e núcleos da base em ambos os hemisférios cerebrais, os quais infiltravam o parênquima (Fig. 1A). No pulmão, havia inúmeras micronodulações pardoavermelhadas e firmes, distribuídas de forma aleatória no órgão (Fig. 1B).

No exame histopatológico do encéfalo, foram observados nódulos multifocais de células neoplásicas fusiformes, com núcleos grandes e alongados e nucléolos evidentes, apresentando cinco a oito figuras de mitose por campo de maior aumento (40x). Essas células estavam dispostas de forma frouxa, formando vacúolos e microcistos, claros e sem conteúdo. As formações microcísticas estavam intercaladas por células neoplásicas fusiformes com núcleo alongado, arranjadas em feixes (Fig. 2A). No parênquima pulmonar, foi observado o mesmo padrão neoplásico descrito no SNC (Fig. 2B). Na imuno-histoquímica, houve marcação fortemente positiva para citoqueratina no encéfalo e no pulmão (Fig. 3A e 3B) e negativa para vimentina (Fig. 4). 


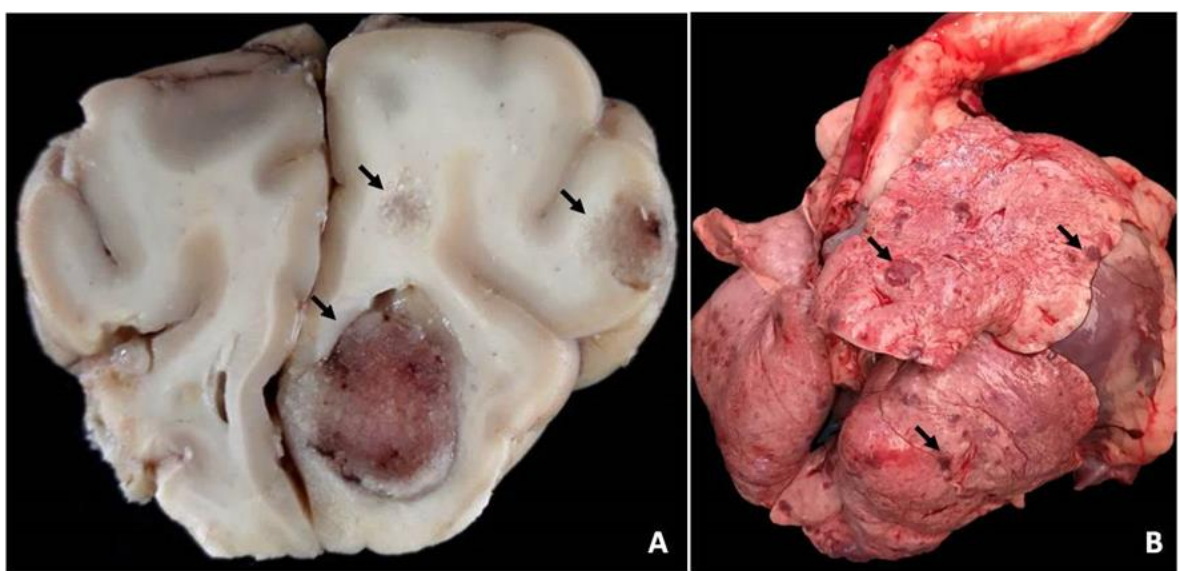

Figura 1A. Encéfalo: massas bem delimitadas pardo-avermelhadas, firmes, de aspecto granular, localizadas no córtex parietal, e núcleos da base (setas). 1B. Pulmão: inúmeros nódulos pardoavermelhados e firmes, distribuídos por todo o parênquima pulmonar (setas).

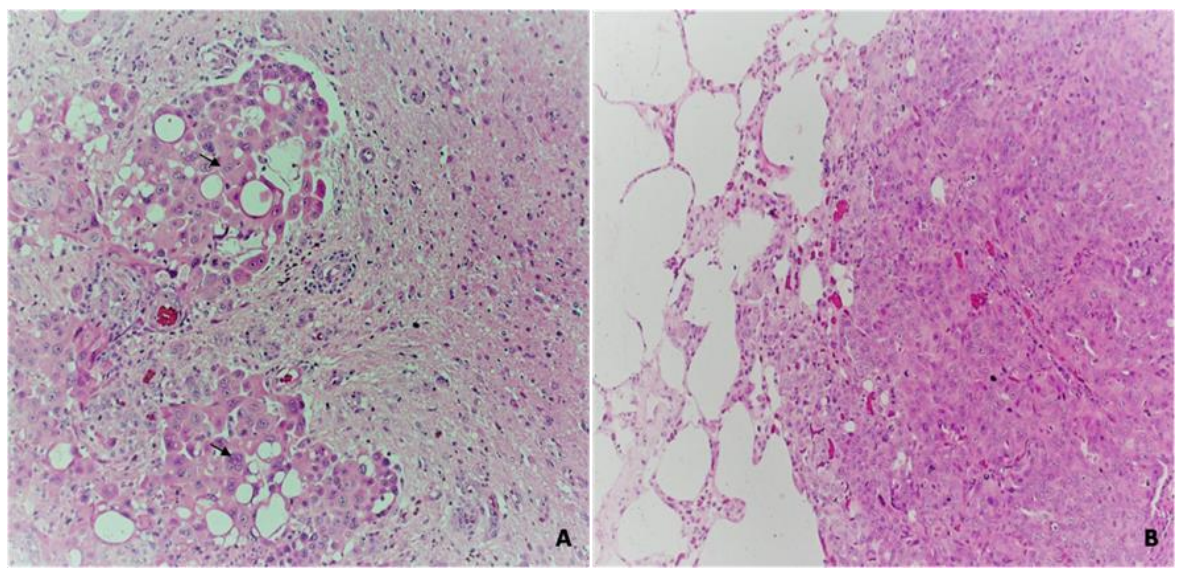

Figura 2A. Encéfalo: meningioma microcístico infiltrando o neurópilo, formando vacúolos e microcistos (setas). HE, obj. 10x. 2B. Pulmão: metástase de meningioma microcístico no parênquima. HE, obj. 4x.
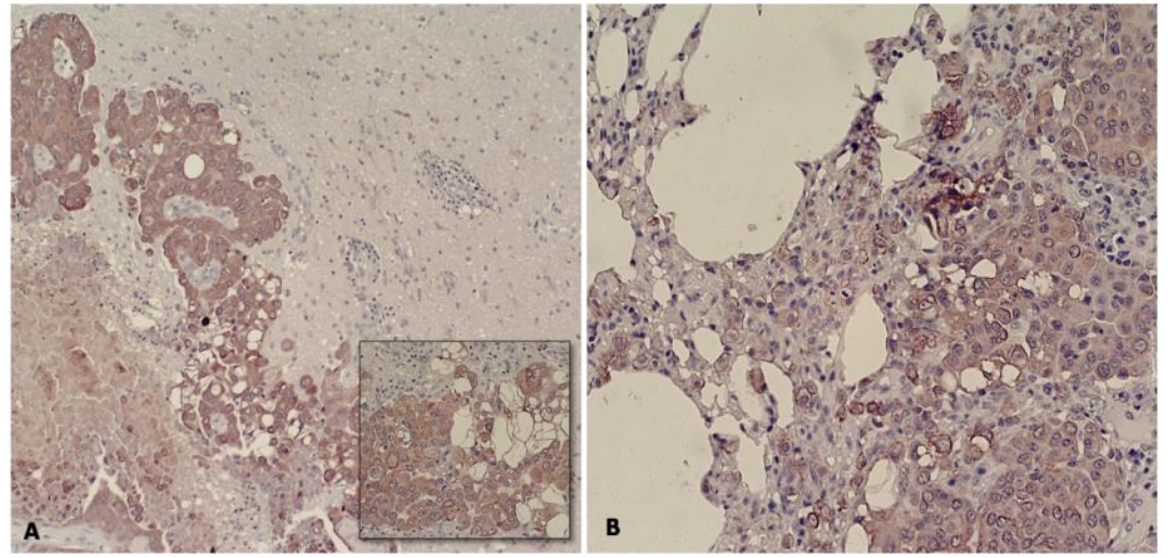

Figura 3A. Encéfalo: imunomarcação positiva para citoqueratina. Imuno-histoquímica citoqueratina (clone AE1/AE3, BioCare Medical), obj. 10x Detalhe. Células neoplásicas com marcação positiva para citoqueratina. Imuno-histoquímica anticitoqueratina, obj. 10x. 3B. Pulmão: imunomarcação positiva para citoqueratina, em metástase pulmonar. Imuno-histoquímica citoqueratina (clone AE1/AE3, BioCare Medical), obj. 10x. 


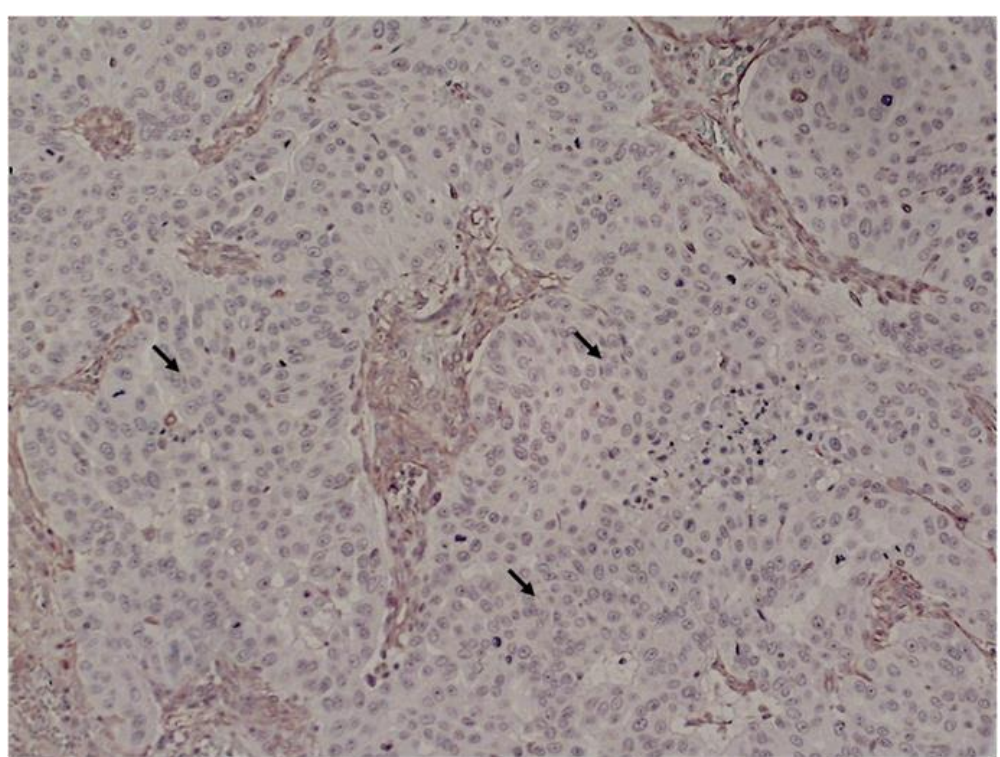

Figura 4. Encéfalo: células neoplásicas com imunomarcação negativa para vimentina (setas). Imunohistoquímica vimentina (clone V9, BioCare Medical), obj. 4x.

\section{DISCUSSÃO}

Em caninos, os meningiomas comumente são tumores benignos, únicos, bem demarcados, granulares e firmes. Localizam-se frequentemente na região do bulbo olfatório e dos lobos frontais, firmemente aderidos à meninge, entretanto também podem ocorrer em outras áreas do SNC (Higgins et al., 2017). No presente caso, havia múltiplas massas distribuídas de forma aleatória nos hemisférios cerebrais, sendo observada, ainda, metástase pulmonar. A presença de múltiplos nódulos no SNC é observada em menos de $10 \%$ dos casos de meningiomas (Marosi et al., 2008; Higgins et al., 2017), sendo rara a ocorrência de metástases (Higgins et al., 2017; Prates et al., 2018). Essa baixa incidência está diretamente relacionada à presença de intercomunicações desmossomais e interdigitações entre as células das meninges, que reduzem a esfoliação celular (Marcasso et al., 2015).

Crises epiléticas e alterações comportamentais, como andar compulsivo e pressão da cabeça contra obstáculos, são os principais sinais clínicos provocados por tumores que acometem a substância branca prosencefálica, os núcleos da base e o diencéfalo (hipotálamo, tálamo e epitálamo) (Santos et al., 2012; Marcasso et al., 2015). No presente caso, apesar da sintomatologia nervosa apresentada pelo canino ter sido sugestiva de neoplasia intracraniana, a confirmação do diagnóstico foi realizada pelos exames histopatológico e imuno-histoquímico, como descrito em outros relatos (Santos et al., 2012; Higgins et al., 2017; Prates et al., 2018).

Os meningiomas podem apresentar diferentes padrões histológicos, sendo essa variabilidade decorrente da origem embriológica mista das meninges, de células neuronais e mesodérmicas (Marcasso et al., 2015). Em cães, os meningiomas transicionais e meningoteliais são os subtipos histológicos observados com maior frequência (Montoliu et al., 2006; Marcasso et al., 2015; Higgins et al., 2017), diferindo do presente relato, que foi classificado como microcístico.

Para o diagnóstico imuno-histoquímico de meningiomas, é utilizada a vimentina, independentemente do subtipo histológico, devido à maior imunorreatividade das células do neoplasma a esse marcador (Higgins et al., 2017). As células normais das meninges apresentam, ainda, características epiteliais, as quais são evidenciadas pela expressão de citoqueratina (Schulman et al., 1992; Kaldrymidou et al., 2001). Nos diferentes subtipos de meningiomas, a imunomarcação positiva para citoqueratina é observada nas regiões com diferenciações microscísticas (Montoliu et al., 2006). No presente relato, 
houve imunomarcação fortemente positiva para citoqueratina e negativa para vimentina, o que permitiu a classificação do subtipo microcístico, visto que, nessa variante, há expressão de citoqueratina (Montoliu et al., 2006). No presente trabalho, a técnica de imunohistoquímica demonstrou ser uma ferramenta essencial para realizar a classificação do padrão histológico de meningioma.

\section{REFERÊNCIAS}

HICKS, J.; PLATT, S.; KENT, M.; HALEY, A. Canine brain tumours: a model for the human disease? Vet. Comp. Oncol., v.15, p.252-272, 2015.

HIGGINS, R.J.; BOLLEN, A.W.; DICKINSON, P.J.; SISÓ-LLONCH, S. Tumors of the nervous system. In: MEUTEN, D.J. (Ed,). Tumors in domestic animals. Ames: John Wiley and Sons, 2017. p.834-891.

KALDRYMIDOU, E.; POLIZOPOULOU, Z.S.; PAPAIOANNOU, N. et al. Papillary meningioma in the dog: a clinic pathological study of two cases. J. Comp. Pathol., v.124, p.227-230, 2001.

MARCASSO, R.A.; MOREIRA, J.R.; VALENTIM, L.G. et al. Meningiomas em cães: aspectos clínicos, histopatológicos e imunohistoquímicos. Pesqui. Vet. Bras., v.35, p.844$852,2015$.

MAROSI, C.; HASSLER, M.; ROESSLER, K. et al. Meningioma. Crit. Rev. Oncol. Hematol., v.67, p.153-171, 2008.
MONTOLIU, P.; ANOR, S.; VIDAL, E.; PUMAROLA, M. Histological and immunohistochemical study of 30 cases of canine meningioma. J. Comp. Pathol., v.135, p.200-207, 2006.

PRATES, K.S.; BIANCHI, M.V.; MELLO, L.S. et al. Spinal cord anaplastic meningioma with extra-neural metastasis in a cat. Cienc. Rural, v.48, p.e20180063, 2018.

SANTOS, R.P.; BECKMANN, D.V.; AIELLO, G. et al. Recuperação funcional de cães paraplégicos com doença do disco intervertebral toraco lombar sem percepção à dor profunda, submetidos ao tratamento cirúrgico: 15 casos (2006-2010). Pesqui. Vet. Bras., v.32, p.243-246, 2012.

SCHULMAN, F.Y.; RIBAS, J.L.; CARPENTER, J.L. et al. Intracranial meningioma with pulmonary metastasis in three dogs. Vet. Pathol., v.29, p.196-202, 1992.

SNYDER, J.M.; SHOFER, F.S.; VAN WINKLE, T.J.; MASSICOTTE, C. Canine intracranial primary neoplasia: 173 cases (19862003). J. Vet. Intern. Med., v.20, p.669-675, 2006.

SONG, R.B.; VITE, C.H.; BRADLEY, C.W.; CROSS, J.R. Postmortem evaluation of 435 cases of intracranial neoplasia in $\operatorname{dog} s$ and relationship of neoplasm with breed, age, and body weight. J. Vet. Intern. Med., v.27, p.11431152, 2013. 